\title{
Cefepime-Induced Non-Convulsive Status Epilepticus in a Patient with Normal Renal Function
}

\section{Case Report}

Journal of Epilepsy Research pISSN 2233-6249 / elSSN 2233-6257

Received April 14, 2016

Accepted June 6, 2016

Corresponding author: Hyeon-Mi Park Department of Neurology, Gachon University Gil Medical Center, 21, Namdong-daero 774 beon-gil,

Namdong-gu, Incheon 21565, Korea

Tel. $+82-32-460-3346$

Fax. $+82-32-460-3344$

E-mail; neurohm@gilhospital.com

\author{
Hyeon-Mi Park* ${ }^{*}$ Young Noh, Ji Won Yang, Dong Hoon Shin, Yeong-Bae Lee \\ Department of Neurology, Gachon University Gil Medical Center, Incheon, Korea
}

Cefepime-induced encephalopathy including nonconvulsive status epilepticus has been known to develop in the patients with renal impairment. However, we report a 74-year-old woman with normal renal function who developed stuporous mental status during cefepime administration. Electroencephalogram (EEG) revealed $2 \mathrm{~Hz}$ rhythmic sharp-and-waves continuously, which suggested nonconvulsive status epilepticus (NCSE). After cefepime discontinuation, clinical symptoms recovered gradually and EEG findings showed only background slowing without epileptiform discharges. Cefepime-induced NCSE could be developed even in the patients with normal renal function, when they are elderly. Therefore, clinicians should be aware of the possibility of cefepime-induced NCSE when prescribing cefepime even to the patients with normal renal function. (2016;6:99-101)

Key words: Cefepime, EEG, Non-convulsive status epilepticus, Renal function

\section{Introduction}

Cefepime is a fourth-generation cephalosporin with broad spectrum coverage of gram-positive and negative bacterial strains. Its potency is higher than many other currently available cephalosporins. As cefepime is excreted via kidney predominantly $(85 \%)$, renal dysfunction may lead to reduction in clearance of cefepime and accumulation in blood flow and cerebrospinal fluid. If the concentration of cefepime reaches the toxic level, it may induce neurotoxicity manifested as myoclonus, altered mental status, and seizure. Thus, it has been reported that cefepime-induced central nervous system (CNS) toxicity, including non-convulsive status epilepticus (NCSE), mainly occurs in patients with impaired renal function. ${ }^{1-4}$ To our knowledge, there is few cases of cefepime-induced NCSE despite normal renal function. ${ }^{5}$ We report a rare case of a 74 -year-old woman with normal renal function, who was diagnosed with NCSE during cefepime administration.

\section{Case}

A 74-year-old woman with history of hypertention was transferred to department of Neurology due to altered mental status developed 1 day ago. Four months ago, she was diagnosed with osteomyelitis at the right mandible and underwent the first surgery to remove sequestrum at the site of infections in the department of dentistry. Postoperatively, however, the patient had a persistent presence of pain. Head and neck computed tomography (CT) scans showed recurrence of inflammation with a pathologic fracture of the surgical site (Fig. 1). Therefore, the patient underwent the second surgery and discharged. At follow-up out-patient clinic, however, recurrence of infection was found at the site of surgical operation again, for which the patient was hospitalized again for third surgery and received imipenem/cilastatin $500 \mathrm{mg}$ every 12 hours. The patient underwent incision and drainage of pus. As an enterococcus species was identi-

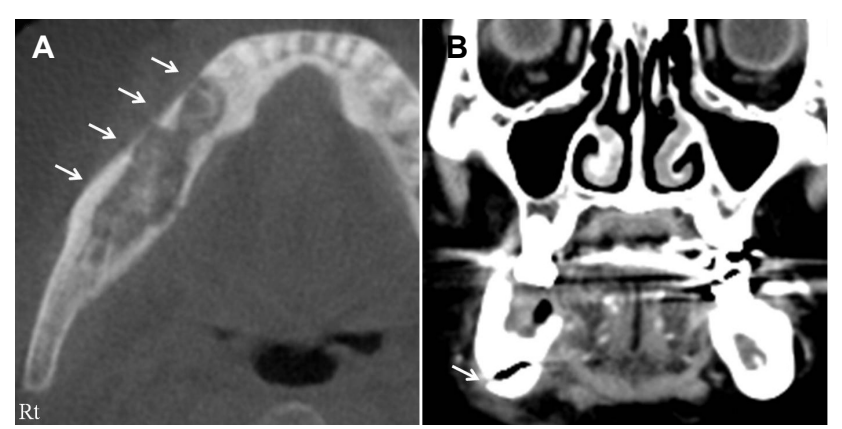

Figure 1. Head and neck CT scans showed oteolytic lesion with cortical erosion in the right mandible (A), and pathologic fracture of the body of the right mandible (B). CT, computed tomography. 
fied, and pus increased in spite of imipenem/cilastatin use, imipenem/cilastatin was replaced with vancomycin. In addition, repeated bacterial culture study identified an Escherichia coli (E. coll). Therefore, the patient received a concomitant use of cefepime $2 \mathrm{~g}$ every 12 hours. The dosage of cefepime was determined considering her renal function. Blood urea nitrogen/creatinine (BUN/Cr) was 12.3/0.7 and creatinine clearance $\left(\mathrm{C}_{\mathrm{Cr}}\right)$ calculated by Modification of Diet in Renal Disease (MDRD) formala was $87 \mathrm{~mL} / \mathrm{min} / 1.73 \mathrm{~m}^{2}$, which suggested normal renal function.

On the 6th day since cefepime administration started, the patient achieved a normalization of vital signs and an improvement of complete blood counts. On day 7 after cefepime used, however, the patient complained of general weakness with somnolence. Moreover, on the 9th day since cefepime administration, the patient presented with stuporous mental status. Then, the patient underwent magnetic resonance imaging (MRI) and a cerebrospinal fluid (CSF) analysis, which showed no abnormal finding. The patient showed stuporous mental status, subtle tonic spasm in both arms and irregular jerky

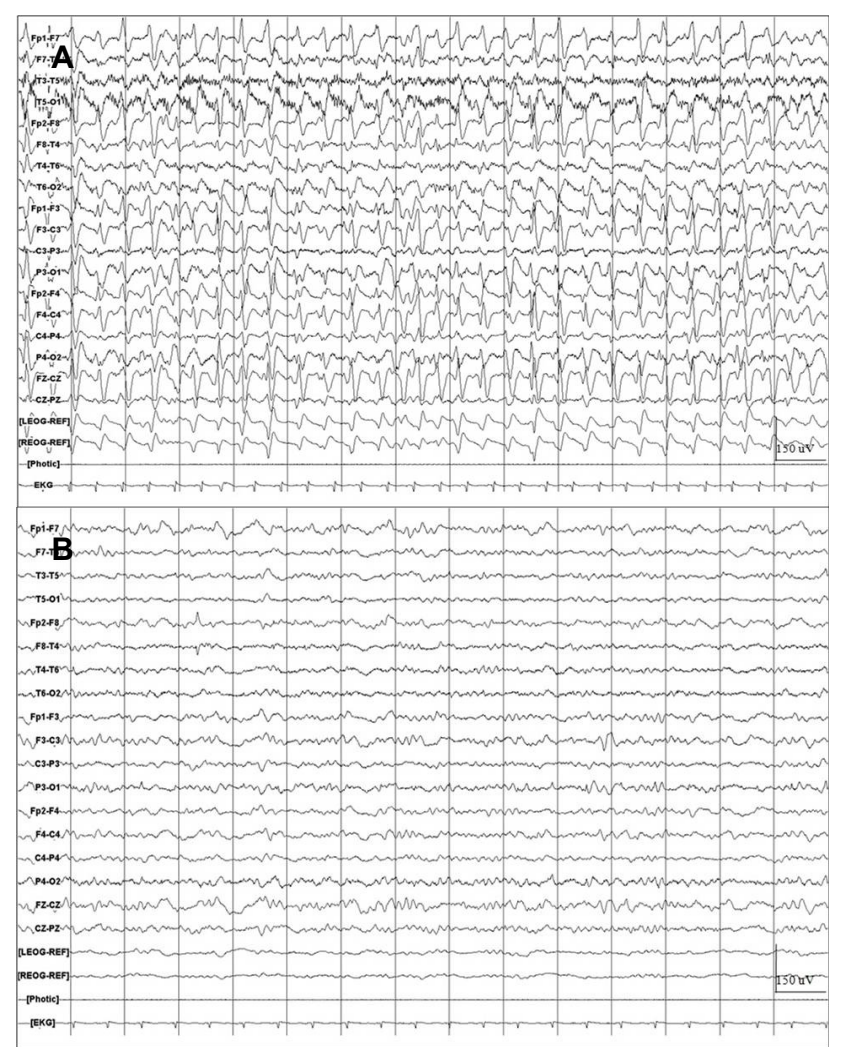

Figure 2. (A) Electroencephalogram (EEG) during cefepime administration showed continuous generalized sharp and slow wave discharges. (B) Next day after the withdrawal of cefepime and the initiation of phenytoin treatment, there were generalized $\theta$ - and $\delta$-slow activities on EEG recordings without any epileptiform discharges. movement like stepping on a pedal in both legs intermittently. We performed electroencephalography (EEG), which showed $2 \mathrm{~Hz}$ rhythmic spike-and-waves continuously. Based on the EEG findings and clinical course, we diagnosed the patient with NCSE (Fig. 2A). As the other etiology causing NCSE was not identified, we suspected cefepime-induced NCSE and cefepime was withdrawed. This was accompanied by the initiation of the intravenous injection of phenytoin 15 $\mathrm{mg} / \mathrm{kg}$. Within 3 days, her mental status was gradually recovered from stupor to drowsiness, and EEG showed only background slowing without epileptiform discharge (Fig. 2B). She recovered to pemorbid status within 4 days after discontinuation of cefepime.

\section{Discussion}

Cefepime-induced neurological complications occur at an approximate incidence of 1-3\%. ${ }^{1,2,6}$ These complications include confusion, delirium, stupor, coma and seizure, which are known to occur within mean period of seven days (range, 1-24 days) after the cefepime treatment. In addition, it has been reported that a mean period of 3 \pm 5 days elapse between the onset of symptoms and the diagnosis of cefepime-induced neurological complications. ${ }^{1,2,6}$ The risk factors of cefepime-induced encephalopathy have been known to be intracranial lesions, advanced age and especially renal failure. ${ }^{1,-9}$ The United State Food and Drug Administration released safety announcement about cefepime and risk of seizure in patients not receiving dosage adjustment for renal impairment. ${ }^{10}$ According to FDA's Adverse Event Reporting System (AERS) database, from the approval of cefepime, in 1996, through February 2012, 59 cases were identified as NCSE during cefepime administration. Renal dysfunction was present in 58 out of 59 patients (in one patient, renal status was unknown). In 56 out of 59 patients, cefepime dose was not appropriately adjusted for renal impairment according to the recommendation in the cefepime label. ${ }^{10}$ As the renal clearance of cefepime would be reduced in patients with renal failure, this may lead to the increased levels of cefepime. Moreover, it is noteworthy that the blood-brain-barrier may be impaired and this would lead to the occurrence of cefepime-induced neurotoxicity in patients with uremic encephalopathy or other types of encephalopathy because it permits the blood-to-brain influx of high-level of cefepime. ${ }^{2,11}$ Still, however, little is known about the exact mechanisms how cefepime-induced neurotoxicity occurs. To explain this, the following mechanisms have been postulated: ${ }^{12,13}$

(1) Cefepime acts as a competitive antagonist of gamma amino- 
butyric acid (GABA)-A and thereby blocks the GABA-A receptor.

(2) Due to the decreased inhibitory activity of GABA, the release of excitatory amino acids is increased or GABA release from nerve terminals is decreased.

(3) The above phenomena lead to the over-excitation of electrical activity of neurons and the occurrence of CNS toxicity such as seizure.

In this recent guideline, the optimal dose of cefepime should be within a narrow range of value as compared with previous ones. Of note, however, cefepime-induced neurotoxicity may also occur even at a controlled, adjusted dose in patients with normal renal function, as shown in our case. Moreover, it would also mandatory to meticulous monitor the creatinine clearance because cefepime-induced nephrotoxicity may occur. Further studies are therefore warranted to determine the appropriate monitoring period. In our case, the patient was monitored for the creatinine clearance at a 3-day interval. On day 7 since cefepime administration, when the patient complaint of general weakness, creatinine clearance was $58 \mathrm{~mL} / \mathrm{min} / 1.73 \mathrm{~m}^{2}$, which was decreased level compared to the creatinine clearance when cefepime administration was started $\left(87 \mathrm{~mL} / \mathrm{min} / 1.73 \mathrm{~m}^{2}\right)$, however, it was still within nearly normal range. Thus the patient took cefepime $2 \mathrm{~g}$ every 12 hours till next day. However, a day after cefepime discontinuation, creatinine clearance revealed her renal function was deteriorated (creatinine clearance $=36 \mathrm{~mL} / \mathrm{min} / 1.73 \mathrm{~m}^{2}$ ). We assumed that renal function on the deteriorating process may contribute to the cefepime-induced NCSE, although creatinine clearance was nearly normal range when cefepime was administrated. Therefore it may be important to monitor the creatinine clearance carefully, detect the change of renal function and adjust cefepime dose according to recommendation in order to prevent cefepime-induced neurotoxicity. It would be necessary to be cautious when the renal function is deteriorating although it is still within normal ranges. It is desirable that serum cefepime levels be measured directly to monitor the optimal dose of cefepime. This deserves further studies because serum cefepime levels are rarely measured in a clinical practice setting. On the other hand, imipenem/cilastatin might have additive effects to the development of encephalopathy, as imipenem has adverse effect of encephalopathy or seizure, especially in the patients with renal dysfunction or brain lesions. ${ }^{14}$ However, for the dosage of imipenem did not exceed the guideline and the patient developed encephalpathy 12 day after the cessation of imipenem, the effect of imipenem would be small, if any.
In conclusion, cefepime-induced NCSE could be developed in the patients with normal renal function, especially they are elderly. Therefore, when clinicians administer cefepime to patients, they should consider cefepime-induced neurotoxicity and check EEG in case of neurologic complication.

\section{References}

1. Martinez-Rodriguez JE, Barriga FJ, Santamaria J, et al. Nonconvulsive status epilepticus associated with cephalosporins in patients with renal failure. Am J Med 2001;111:115-9.

2. Abanades $S$, Nolla J, Rodríguez-Campello A, Pedro C, Valls A, Farré M. Reversible coma secondary to cefepime neurotoxicity. Ann Pharmacother 2004;38:606-8.

3. Lin $C M$, Chen $Y M$, Po $H L$, Hseuh $\mathrm{H}$. Acute neurological deficits caused by cefipime: a case report and review of literature. Acta Neurol Taiwan 2006;15:269-72.

4. Jallon $P$, Fankhauser $L$, Du Pasquier $R$, et al. Severe but reversible encephalopathy associated with cefepime. Neurophysio/ Clin 2000;30:383-6.

5. Maganti $R$, Jolin $D$, Rishi $D$, Biswas A. Nonconvulsive status epilepticus due to cefepime in a patient with normal renal function. Epilepsy Behav 2006;8:312-4.

6. Dakdouki GK, Al-Awar GN. Cefepime-induced encephalopathy. Int J Infect Dis 2004;8:59-61.

7. Thabet F, Al Maghrabi M, Al Barraq A, Tabarki B. Cefepime-induced nonconvulsive status epilepticus: case report and review. Neurocrit Care 2009; 10:347-51.

8. Garces EO, Andrade de Anzambuja MF, da Silva D, Bragatti JA, Jacoby T, Saldanha Thomé $F$. Renal failure is a risk factor for cefepime-induced encephalopathy. J Nephrol 2008;21:526-34.

9. Chow KM, Hui AC, Szeto CC. Neurotoxicity induced by beta-lactam antibiotics: from bench to bedside. Eur I Clin Microbiol Infect Dis 2005;24:649-53.

10. FDA Drug Safety Communication. Cefepime and risk of seizure in patients not receiving dosage adjustments for kidney impairment [Internet]. Silver Spring (MD): Food and Drug Administration, [cited 2016 Apr 10]. Available at: http://uww.fda.gov/Drugs/DrugSafety/ucm309661.htm.

11. Barbey F, Bugnon D, Wauters JP. Severe neurotoxicity of cefepime in uremic patients. Ann Intern Med 2001;135:1011.

12. Curtis DR, Game CJ, Johnston GA, McCulloch RM, MacLachlan RM. Convulsive action of penicillin. Brain Res 1972;43:242-5.

13. De Sarro A, Ammendola D, Zappala M, Grasso S, De Sarro GB. Relationship between structure and convulsant properties of some beta-lactam antibiotics following intracerebroventricular microinjection in rats. Antimicrob Agents Chemother 1995;39:232-7.

14. Sutter R, Ruegg $S$, Tschudin-Sutter $S$. Seizures as adverse events of antibiotic drugs: A systematic review. Neurology 2015;85:1332-41. 